Portland State University

PDXScholar

Computer Science Faculty Publications and

Presentations

Computer Science

2-18-2014

\title{
Damage Spreading in Spatial and Small-world Random Boolean Networks
}

\author{
Qiming Lu \\ Fermi National Accelerator Laboratory \\ Christof Teuscher \\ Portland State University, teuscher@pdx.edu
}

Follow this and additional works at: https://pdxscholar.library.pdx.edu/compsci_fac

Part of the Computer Sciences Commons

Let us know how access to this document benefits you.

\section{Citation Details}

Lu, Qiming, and Christof Teuscher. "Damage spreading in spatial and small-world random Boolean networks." Physical Review E 89.2 (2014): 022806.

This Article is brought to you for free and open access. It has been accepted for inclusion in Computer Science Faculty Publications and Presentations by an authorized administrator of PDXScholar. Please contact us if we can make this document more accessible: pdxscholar@pdx.edu. 


\title{
Damage spreading in spatial and small-world random Boolean networks
}

\author{
Qiming $\mathrm{Lu}^{1, *}$ and Christof Teuscher ${ }^{2, \dagger}$ \\ ${ }^{1}$ Scientific Computing Division, Fermi National Accelerator Laboratory, Batavia, Illinois 60510-5011, USA \\ ${ }^{2}$ Department of Electrical and Computer Engineering (ECE), Portland State University, P.O. Box 751, Portland, Oregon 97207-0751, USA
}

(Received 23 September 2013; published 18 February 2014)

\begin{abstract}
The study of the response of complex dynamical social, biological, or technological networks to external perturbations has numerous applications. Random Boolean networks (RBNs) are commonly used as a simple generic model for certain dynamics of complex systems. Traditionally, RBNs are interconnected randomly and without considering any spatial extension and arrangement of the links and nodes. However, most realworld networks are spatially extended and arranged with regular, power-law, small-world, or other nonrandom connections. Here we explore the RBN network topology between extreme local connections, random smallworld, and pure random networks, and study the damage spreading with small perturbations. We find that spatially local connections change the scaling of the Hamming distance at very low connectivities $(\bar{K} \ll 1)$ and that the critical connectivity of stability $K_{s}$ changes compared to random networks. At higher $\bar{K}$, this scaling remains unchanged. We also show that the Hamming distance of spatially local networks scales with a power law as the system size $N$ increases, but with a different exponent for local and small-world networks. The scaling arguments for small-world networks are obtained with respect to the system sizes and strength of spatially local connections. We further investigate the wiring cost of the networks. From an engineering perspective, our new findings provide the key design trade-offs between damage spreading (robustness), the network's wiring cost, and the network's communication characteristics.
\end{abstract}

DOI: 10.1103/PhysRevE.89.022806

PACS number(s): 05.65.+b, 05.45.-a, 89.75.-k

\section{INTRODUCTION}

The robustness against failures, the wiring cost, and the communication characteristics are key measures of most complex, finite-size real-world networks. For example, the electrical power grid needs to be robust against a variety of failures, minimize the wiring cost, and minimize the transmission losses. Similarly, the neural circuitry in the human brain requires efficient signal transmission and robustness against damage while being constrained in volume.

In this article, we use random Boolean networks (RBNs) as a simple model to study the (1) robustness, i.e., the damage spreading, (2) the wiring cost, and (3) the communication characteristics as a function of different network topologies (local, small-world, random), different connectivities $\bar{K}$, and different network sizes $N$. More generally speaking, this allows us to answer the question of how much and what type of interconnectivity a complex network - in our case RBNs - needs in order to satisfy given restrictions on the robustness against certain types of failure, the (wiring) cost, and the (communication) efficiency. The work presented here extends previous work by Rohlf et al. [1] to new network topologies, which are more biologically plausible, such as small-world topologies [2].

RBNs were originally introduced by Kauffman as simplified models of gene regulation networks [3,4]. In its simplest form, an RBN is a discrete dynamical system, also called an $N K$ network (or model), composed of $N$ automata (or nodes), each of which receives inputs from $K$ (either exact or average) randomly chosen other automata. Each automaton is a Boolean variable with two possible states $\{0,1\}$, and the dynamics is

\footnotetext{
•qlu@fnal.gov

†teuscher@pdx.edu
}

such that

$$
\text { F : }\{0,1\}^{N} \mapsto\{0,1\}^{N} \text {, }
$$

where $\mathbf{F}=\left(f_{1}, \ldots, f_{i}, \ldots, f_{N}\right)$, and each $f_{i}$ is represented by a look-up table of $K_{i}$ inputs randomly chosen from the set of $N$ automata. Initially, $K_{i}$ neighbors and a look-up table are assigned to each automaton at random.

$$
f_{i}:\{0,1\}^{K_{i}} \mapsto\{0,1\} .
$$

An automaton state $\sigma_{i}^{t} \in\{0,1\}$ is updated using its corresponding Boolean function:

$$
\sigma_{i}^{t+1}=f_{i}\left(x_{i_{1}}^{t}, x_{i_{2}}^{t}, \ldots, x_{i_{X_{i}}}^{t}\right) .
$$

We randomly initialize the states of the automata (initial condition of the RBN). The automata are updated synchronously using their corresponding Boolean functions.

$$
\sigma^{t+1}=\mathbf{F}\left(\sigma^{t}\right) \text {. }
$$

In the thermodynamic limit, RBNs exhibit a dynamical order-disorder transition at a sparse critical connectivity $\boldsymbol{K}_{c}$ [5]. For a finite system size $N$, the dynamics of RBNs converge to periodic attractors after a finite number of updates. At $\boldsymbol{K}_{c}$, the phase space structure in terms of attractor periods [6], the number of different attractors [7], and the distribution of basins of attraction [8] are complex, showing many properties reminiscent of biological networks [4].

The study of the response of complex dynamical networks to external perturbations, also referred to as damage, has numerous applications, e.g., the spreading of disease through a population $[9,10]$, the spreading of a computer virus on the internet [11], failure propagation in power grids [12], the perturbation of gene expression patterns in a cell due to mutations [13], or the intermittent stationary state in economic decision networks triggered by the mutation of strategy from 
a few individual agents [14]. Mean-field approaches, e.g., the annealed approximation (AA) introduced by Derrida and Pomeau [5], allow for an analytical treatment of damage spreading and exact determination of the critical connectivity $K_{c}$ under various constraints $[15,16]$. However, these approximations rely on the assumption that $N \rightarrow \infty$, which, for an application to real-world problems, is often an irrelevant limit. A number of studies $[17,18]$ have recently focused on the finite-size scaling of (un)frozen and/or relevant nodes in RBN with respect to $N$ with the goal to go beyond the annealed approximation. Evolved small-world and scale-free networks were investigated for the density and the synchronization task with regard to performance and robustness in [19]. Their work solely focuses on these two tasks and does not consider scaling arguments. Only a few studies, however, consider finite-size scaling of damage spreading in RBNs $[1,13,20]$. Of particular interest is the "sparse percolation (SP) limit" [20], where the initial perturbation size $d(0)$ does not scale up with the network size $N$, i.e., the relative size of perturbations tends to zero for large $N$. Rohlf et al. [1] have identified a new characteristic connectivity $K_{s}$ for RBNs, at which the average number of damaged nodes $\bar{d}$, after a large number of dynamical updates, is independent of $N$. This limit is particularly relevant to information and damage propagation in many technological and natural networks. The work in this article extends these new findings and systematically studies damage spreading in RBNs as a function of new network topologies, namely, local and small-world, different connectivities $\bar{K}$, and different network sizes $N$.

\section{DAMAGE SPREADING}

For our purpose, we measure the expected damage $\bar{d}$ as the Hamming distance between two different initial system configurations after a large number of system updates $T$. The randomly chosen initial conditions differ by one bit, i.e., the damage size is 1 . As introduced in [1], let $\mathcal{N}$ be a randomly sampled set (ensemble) of $z_{N}$ networks with average degree $\bar{K}, \mathcal{I}_{n}$ a set of $z_{I}$ random initial conditions tested on network $n$, and $\mathcal{I}_{n}^{\prime}$ a set of $z_{I}$ random initial conditions differing in one randomly chosen bit from these initial conditions. Then we have

$$
\bar{d}=\frac{1}{z_{N} z_{I}} \sum_{\substack{n=1 \\ \mathcal{N}_{n} \in \mathcal{N}}}^{z_{N}} \sum_{\substack{i=1 \\ \vec{\sigma}_{i} \in \mathcal{I}_{n}, \vec{\sigma}_{i}^{\prime} \in \mathcal{I}_{n}^{\prime}}}^{z_{I}} d_{i}^{n}(T),
$$

where $d_{i}^{n}(T)$ is the measured Hamming distance after $T$ system updates. Rohlf et al. [1] have shown that there exists a characteristic connectivity $K_{s}$, at which the average number of damaged nodes $\bar{d}$, after a large number of dynamical updates, is independent of $N$.

In a given network, the nodes can be classified according to their response to the network dynamics (e.g., see [17,18]). This classification allows one to better explain the global network behavior with respect to external perturbations.

A set of nodes is said to be part of the frozen component (or frozen core) if each node's output is constant regardless of its inputs. The states of these nodes remain constant on every attractor, so that external perturbations cannot spread into the frozen component. The frozen core therefore does not contribute to the spreading of the damage. The irrelevant nodes (or irrelevant component) are the nodes whose outputs may change, but their outputs are only connected to either frozen or other irrelevant nodes. Again, these nodes do not participate in the damage spreading. The remaining set of nodes is the relevant nodes (or relevant component). Their state changes and each relevant node is connected to at least another relevant node. As their name suggests, the relevant nodes are the crucial ones, which determine the number and the period of attractors in a given network. For our purpose, studying the scaling behavior of the Hamming distance is important for the study of damage spreading because the Hamming distance between the damaged and the undamaged network can be viewed as a quantitative measure of the distance between the two different attractors the networks settle in.

In this article, we use three exemplary types of network topologies: (1) random, (2) spatially local, and (3) small world. In the following we will describe the models we used to create each of these network topologies and what the relevant parameters are. For more details see the text in the next three sections (II A-II C). Note that in all of these network topologies, the links are directed, self-loops are allowed, and multiple links between the same pair of nodes are excluded.

a. Random topology. Each of the $N$ nodes has a uniform probability to be connected to any other node in the network. The average connectivity is $\bar{K}$. This topology corresponds to the original $N K$ model proposed by Kauffman [3].

b. Spatially local topology. $N$ nodes are uniformly and randomly distributed in a unit $d$-dimensional spatial area (nonperiodical). Each node randomly connects to its nearest neighbors (including itself) until the designated $\bar{K}$ is reached. This network topology can be classified as a spatial graph. In the limit of small $\bar{K}(\bar{K} \ll N)$, such a $d$-dimensional, spatially local network has an average path length of $\sim N^{1 / d}[21]$, which is similar to a $d$-dimensional regular lattice [22].

c. Small-world topology. Starting from two-dimensional (2D) spatially local networks as described above, we apply a rewiring method to obtain a small-world network topology. The source of every existing link will be rewired with probability $p$ to a randomly chosen node in the network. Thus, when $p \rightarrow 0$, we obtain the original spatially local network, while for $p \rightarrow 1$ we obtain a random graph as described above.

\section{A. RBNs with a random network topology}

Rohlf et al. [1] have systematically investigated damage spreading, i.e., the evolution of the Hamming distance $\bar{d}_{R}$, of random Boolean networks at the SP limit. By using finite-size scaling, they found a new characteristic connectivity $K_{S}=$ 1.875 at which the damage spreading is independent of the system size $N$.

In the limit of a small average degree $\bar{K} \rightarrow 0$, the initial perturbation persists only when the damage hits nodes that are in loops of length two or that have self-connections. For a random network topology, the probability of generating such loops scales with $\boldsymbol{P}_{\text {loop }} \sim 1 / N^{2}$, where $N$ is the system size. Thus, the Hamming distance is proportional to the number of simple loops, $\bar{d}_{R} \sim P_{\text {loop }} \bar{K} N \sim N^{-1}$. For large $\bar{K}$, the relevant component grows comparable with the system size, so the initial damage now percolates through the entire network, and 

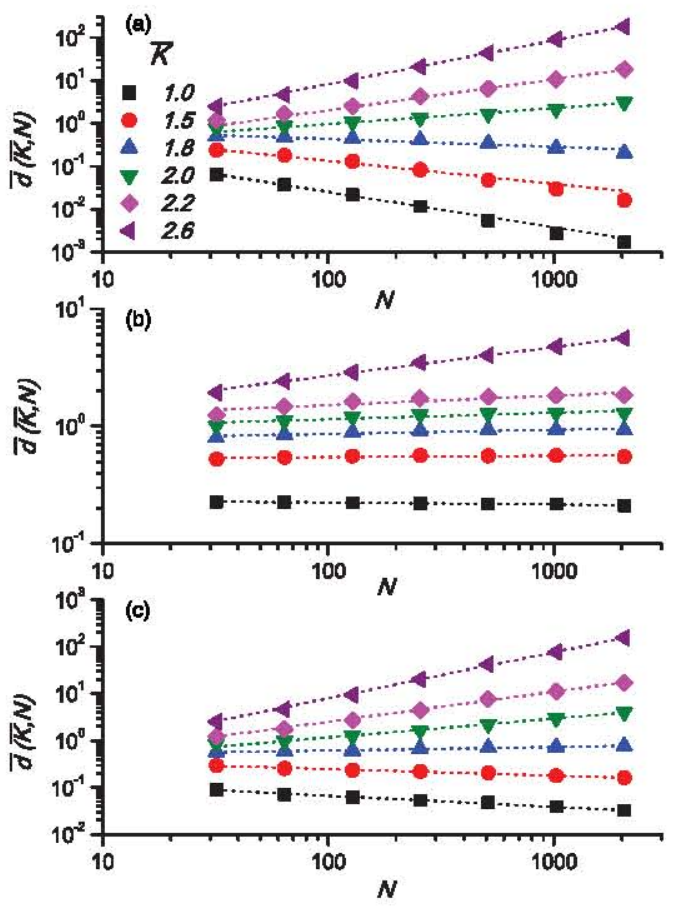

FIG. 1. (Color online) The Hamming distance $\bar{d}$ as a function of the system size $N$ for (a) random networks, (b) networks with spatially local connections, and (c) small-world networks, for different $\bar{K}$. $\bar{K}$ takes the values $2.6,2.2,2.0,1.8,1.5$, and 1.0, from top to bottom. The data for the random networks confirms the data as first presented in [1]. Averaged over 10000 randomly generated networks and 100 random initial configurations for each value of $\bar{K} . T=1000$ system updates, initial damage size $d(0)=1$.

we have $\bar{d}_{R} \sim N$. For arbitrary $\bar{K}$, the Hamming distance $\bar{d}_{R}$ scales as follows [1]:

$$
\bar{d}_{R}(\bar{K}, N) \sim a(\bar{K}) N^{\gamma_{K}(\bar{K})},
$$

where $\gamma_{R} \rightarrow-1$ at $\bar{K} \rightarrow 0$ and $\gamma_{R} \rightarrow 1$ at large $\bar{K}$. At criticality (i.e., $K=2$ ), the asymptotic dynamics are determined entirely by the relevant component, which scales as $n_{r} \sim N^{1 / 3}$ [17], thus $\gamma_{R}\left(K_{c}\right) \simeq 1 / 3$. As already seen above, at $\bar{K}=K_{s}=1.875$ we have $\gamma_{R}\left(K_{s}\right)=0$, and the Hamming distance $\bar{d}$ is independent of the system size $N$ [1]. This means that at the "critical connectivity of stability" $K_{s}$, the damage caused by initial perturbations is confined at a finite level (i.e., the proportion of damage goes to zero as $N \rightarrow \infty$ ), regardless of the system size $N$.

Figure 1 shows the power-law dependence of the Hamming distance $\bar{d}$ as a function of the system size $N$ for multiple $\bar{K}$ and for our three types of random network classes. $\gamma_{R}$ as a function of the average degree $\bar{K}$ is shown in Fig. 2.

\section{B. RBNs with spatially local connections}

Many real-world networks are spatially extended and have a more structured interconnect topology than pure random networks have. Such networks are commonly called complex networks. Spatial networks with local connections only, such as regular grids, have a large average path length $\left(l \sim N^{1 / d}\right)$ and are highly clustered. In this section, we look at the

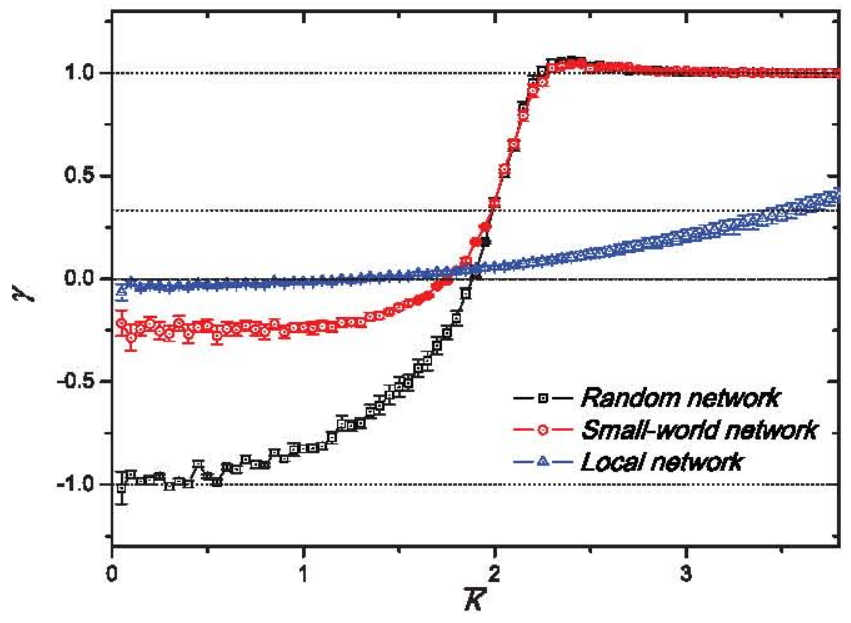

FIG. 2. (Color online) Scaling exponents $\gamma(\bar{K})$ as a function of $\bar{K}$ for random networks (open squares), networks with local connections (open triangles), and small-world networks (open circles). This figure is obtained from the best fit of the data of Fig. 1 using Eqs. (6), (7), and (10). The data for the random networks confirms the data as first presented in [1].

dynamics of spatial RBNs with local connections only. The underlying network structure is constructed based on the model of uniform spatial graphs [21], in which vertices may connect uniformly at random to other vertices within a spatial distance $l_{c}$ in $\mathbb{R}^{d}$. We do this as follows: $N$ nodes are randomly distributed in a $d$-dimensional space (only $d=2$ will be considered here); we then randomly pick a pair of nodes $u, v$ and create edge $(u, v)$ if the spatial distance is within the cut-off distance $l_{c}$, disallowing repeated edges. This procedure is repeated until the required average degree $\bar{K}$ is reached. For a small cutoff distance, or any finite cutoff when $N \rightarrow \infty$, e.g., $l_{c} \sim O(1)$, the characteristic path length remains similar to that of $d$-dimensional regular lattices [21]. Establishing links as a function of the distance, was also considered by $[23,24]$.

For very large $\bar{K}$, the system is in the chaotic regime and any initial damage quickly percolates through the network. Thus, the damage is only bounded by the system size $N$, which gives us $\bar{d}_{L} \sim N^{1}$. In the limit of $\bar{K} \rightarrow 0$, nonzero damage can emerge only when the initial perturbation hits a short loop of oscillating nodes. Let us assume we have a single connection from node $A$ to node $B(A \rightarrow B)$. In order to finish a simple loop between $A$ and $B$, we need to first select node $B$ as the starting point, which has a probability of about $\sim 1 / N$. The probability to pick $A$ as a neighboring node from $B$ to close the loop is $\sim 1 / n_{B}$, where $n_{B}$ is the possible number of $B$ 's local neighbors. For a purely local network, $n_{B} \ll N$. In a network of extreme local connections, the probability of forming simple oscillating loops scales with $P_{\text {loop }} \sim 1 /\left(n_{B} N\right) \sim N^{-1}$. The number of such loops scales with $\sim P_{\text {loop }} \bar{K} N \sim$ const, and is thus independent of the system size $N$. We expect to see coinciding Hamming distances at low $\bar{K}$ for different system sizes $N$ on extremely local networks. This remains valid until the network reaches the percolation threshold where segregated simple loops become connected and a giant cluster emerges. Furthermore, compared to random networks, the local connections lowered the probability of 
forming the relevant component at criticality because each relevant node needs to be controlled by another relevant node. We thus expect that the damage increases slower compared to random networks. In particular, the exponent is smaller than $1 / 3$ at $K_{c}$ because $\gamma=1 / 3$ at $K_{c}$ for random networks [1].

Figure 1(b) shows the Hamming distance as a function of the system size $N$ for different connectivities $\bar{K}$. As one can see, for small $\bar{K}$, the damage remains constant as $N$ increases, whereas for large $\bar{K}$ (above the percolation limit) the damage spreading increases with the system size $N$ according to a power law. We therefore have

$$
\bar{d}_{L}(\bar{K}, N) \sim a(\bar{K}) N^{\gamma_{L}(\bar{K})},
$$

where $\gamma_{L} \rightarrow 0$ at $\bar{K} \rightarrow 0$, and $\gamma_{L} \rightarrow 1$ for large $\bar{K}$. If we do a best fit for the data as shown in Fig. 1(b) using Eqs. (6) and (7), we obtain $\gamma_{L}$ as a function of $\bar{K}$. This is shown in Fig. 2.

Finally, Fig. 3(d) shows the average Hamming distance for an initial damage size of one for local networks with different system sizes $N$. As one can see, all curves coincide below the percolation threshold. This confirms again our assumption of the scaling behavior for low $\bar{K}$.

\section{RBNs with a small-world topology}

Both purely random and purely local networks are extreme network topologies. Many biological, technological, and social networks lie somewhere between these two extremes and are categorized as "small-world networks" [25]. Small-world networks typically exhibit a number of advantages over locally connected networks, such as a short average path length, synchronizability, and improved robustness against certain types of failures [22]. It is therefore of fundamental interest to study the damage spreading in RBN networks with a small-world interconnect topology.

Starting from the 2D uniform spatial graph we have used above for the locally interconnected network, we apply a simple rewiring strategy to construct a small-world network. Each existing connection in the uniform spatial graph is rewired with probability $p$ to a randomly chosen node. Thus, a fraction of $p$ links in the network are random long-range links, or small-world links, while the remaining fraction of $q=1-p$ links are local links connecting geometrically local neighbors. We will use $q$ as the main parameter to represent the "strength" of the local connections. Note that for the extreme case of complete random spatial networks, by definition the density of the local connections is $\sim 1 / N$. Combined with the system size $N, N q$ is approximately the number of nodes that have a local connection (at the sparse percolation limit). For $N q \sim 1$ the network is in the random regime (see Sec II A); and for $N q \gg 1$ we obtain a spatially local network (see Sec. II B).

We will now use a similar scaling approach for small-world RBNs as presented above for local and random networks. Again, at very large $\bar{K}$, the damage will only be bounded by the system size, thus $\bar{d}_{\text {sw }} \sim N^{1}$. But for $\bar{K} \rightarrow 0$, the network is now composed of both local and random (longer range) connections and the probability of forming simple loops thus scales differently. Let us assume we have a local link that has
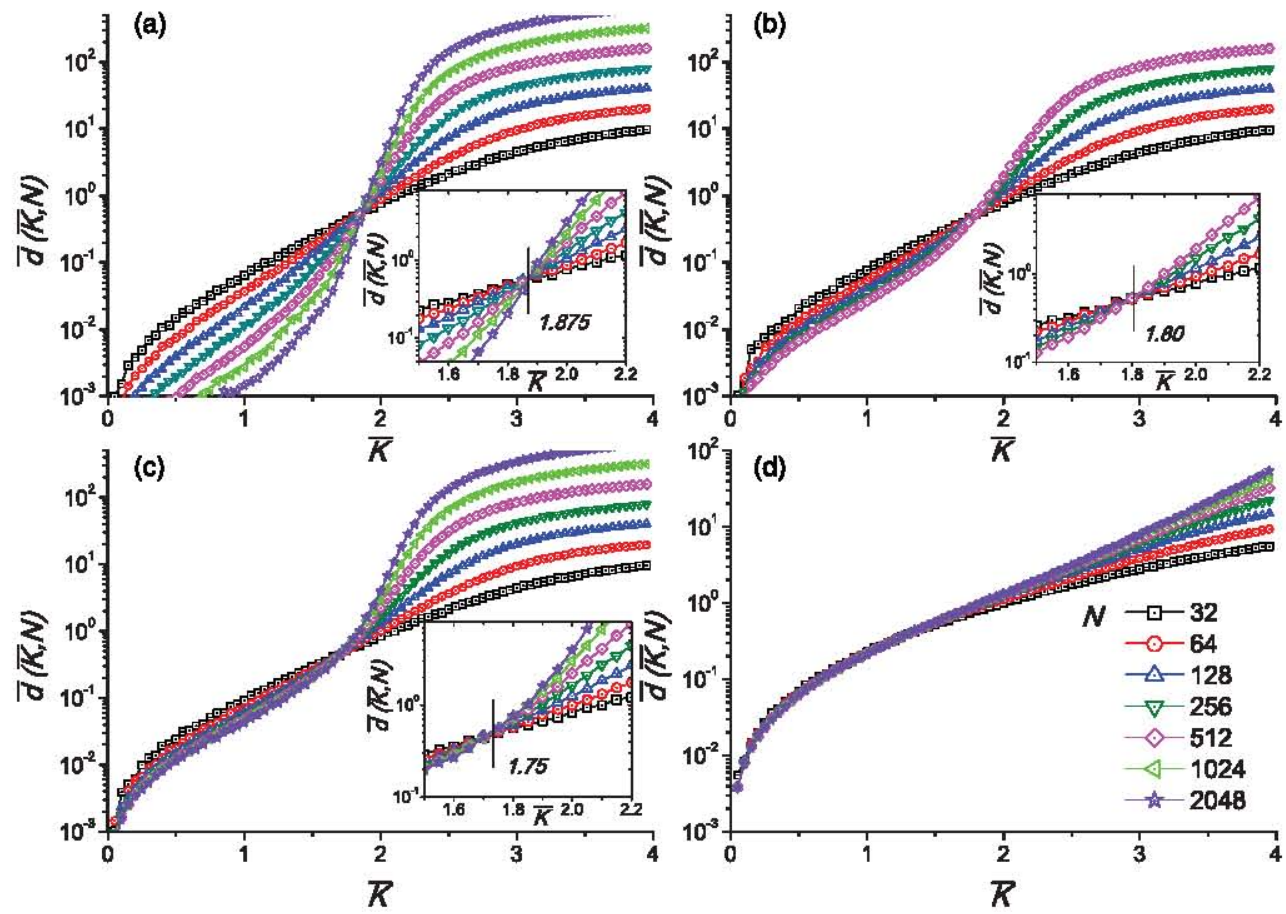

FIG. 3. (Color online) Average Hamming distance (damage) $\bar{d}$ after 200 system updates, averaged over 10000 randomly generated networks and 100 random initial configurations for each value of $\bar{K}$. The initial damage size is one. Network topologies: (a) random networks $(p=1.0$, $q=0$ ), (b) small-world network with $p=0.9$ ( $q=0.1$ ), (c) small-world networks with $p=0.8(q=0.2)$, and (d) networks with completely local connections $(p=0, q=1.0$ ). (a)-(c) suggest that all curves of random and small-world networks for different $N$ approximately intersect in a characteristic point $K_{s} . K_{s}$ moves toward small $\bar{K}$ as the fraction of local connections increases $\left[K_{s} \approx 1.875\right.$ in (a), $K_{s} \approx 1.80$ in (b), and $K_{s} \approx 1.75$ in (c)]. For complete local networks all curves coincide below the percolation threshold independently of $N$. 
already been connected $(A \rightarrow B)$. The probability of having such a local link is $q$, and to complete a simple loop that contains this local connection, we first need to pick node $B$ with probability $1 / N$. Node $B$ will then establish connections again with his local neighbors with probability $q$, and finally choose node $A$ to finish the loop with probability $1 / n_{B}$. Thus, the final probability of having a simple loop in this case scales with $P_{\text {loop } L} \sim q^{2} / N$. Similarly the probability of generating a simple loop involving random long-range links is $P_{\text {loop } R} \sim p^{2} / N^{2}$. We compare these two probabilities by dividing one by another:

$$
\frac{P_{\operatorname{loop} L}}{P_{\operatorname{loop} R}}=\frac{q^{2}}{N} / \frac{p^{2}}{N^{2}}=(N q) \frac{q}{p^{2}} .
$$

In the spatially local network limit $(N q \gg 1), P_{\text {loop } L}$ is the leading term and the scaling follows Eq. (7). In the random network limit ( $q \rightarrow 0$ and $N q \sim 1$ ), $P_{\text {loop } R}$ dominates and the scaling follows Eq. (6). However, when the network is in the small-world regime, $P_{\text {loop } L}$ and $P_{\text {loop } R}$ become comparable. With some corrections, we therefore have

$$
P_{\text {loop SW }}=P_{\text {loop } L}+P_{\text {loop } R}=\frac{q^{2}}{N}+\frac{p^{2}}{N^{2}} \simeq \frac{1}{N^{\beta}},
$$

where $\beta$ is somewhere between 1 and 2 and depends on the value of $N q$. The damage spreading scales therefore with $\bar{d}_{\mathrm{sw}} \sim N^{1-\beta}$. And for general $\bar{K}$, we obtain

$$
\bar{d}_{\mathrm{sw}}(\bar{K}, N) \sim a(\bar{K}) N^{\gamma_{\mathrm{sw}}(\bar{K})},
$$

where $\gamma_{\mathrm{SW}}$ is somewhere between $1-\beta$ and 1 . Figure 2 shows $\gamma_{R}, \gamma_{L}$, and $\gamma_{\mathrm{SW}}$. As one can see, $\gamma_{\mathrm{SW}}$ goes from $1-\beta$, which is below zero, to 1 as $\bar{K}$ increases. In addition, the critical connectivity $\bar{K}$, where $\gamma_{\mathrm{SW}}=0$, is different from that of random networks and depends on $q$. In random networks this point is defined as the critical degree of stability $K_{s}$ [1]. Our results show that the introduction of local connections in random networks changes $K_{s}$ toward lower $\bar{K}$. As we have seen above, in extreme local networks, $K_{s}$ is undefined because the Hamming distance for different system sizes $N$ simply coincide below the percolation threshold. Figure 3(c) shows the deviation of $K_{s}$ from the observed value $K_{s}=1.875$ for random networks.

\section{Scaling of damage spreading in random small-world RBNs}

While Eqs. (6) and (7) provide the scaling of the Hamming distance for random and local networks, we are interested in this section in how $q$ (i.e., the fraction of local links) affects the damage spreading.

Figure 4 shows the Hamming distance as a function of $q$. For sufficiently large $N q$, i.e., close to the local network limit, we assume (see Fig. 4) that the Hamming distance approaches an asymptotic power law $\bar{d}_{\mathrm{SW}} \sim q^{\alpha(\bar{K})}$. On the other hand, the Hamming distance also depends on the system size with $\bar{d}_{\mathrm{Sw}} \sim$ $N^{\text {sw }(\bar{K})}$ [see Eq. (10)]. Thus, in the small-world regime (close to the local network limit) the Hamming distance depends on both the system size and the density of local (random) connections:

$$
\bar{d}_{\mathrm{Sw}}(q, \bar{K}, N) \sim q^{\alpha(\bar{K})} N^{\gamma_{\mathrm{sw}}(\bar{K})} .
$$

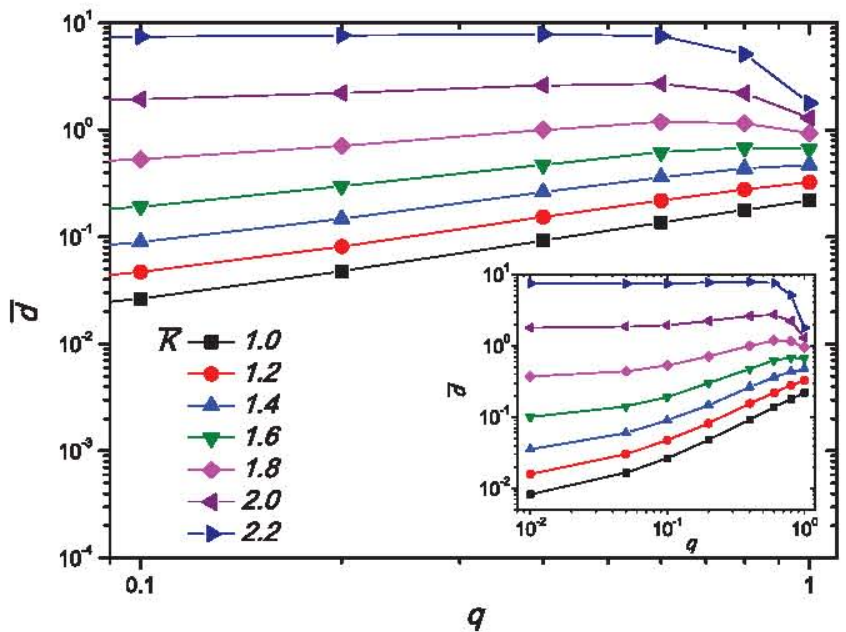

FIG. 4. (Color online) Hamming distance $\bar{d}$ for different average degrees $\bar{K}$ as a function of the density of local connections $q$. The inset shows $\bar{d}$ in the range of random networks $(q \rightarrow 0)$ to local networks ( $q \rightarrow 1)$. The different curves range from (top to bottom) $\bar{K}=2.2$ to $\bar{K}=1.02$ with an interval of 0.2 .

When $q \rightarrow 1, \gamma_{\mathrm{SW}}(\bar{K}) \rightarrow \gamma_{L}(\bar{K})$, so $\bar{d}_{\mathrm{SW}} \rightarrow \bar{d}_{L}$. While in the random network limit $(N q \sim 1), \bar{d}$ only depends on $N, \bar{d}_{R} \sim$ $N^{\gamma_{R}(\bar{K})}$ with $\gamma_{R}(\bar{K})$ as illustrated in Fig. 2. To connect the above two cases and to capture the finite-size behavior in the small-world regime, one can construct the full scaling behavior of $\bar{d}_{\mathrm{Sw}}(q, \bar{K}, N)$ :

$$
\bar{d}_{\mathrm{SW}}(q, \bar{K}, N) \sim q^{\alpha(\bar{K})} N^{\gamma_{\mathrm{Sw}}(\bar{K})} f(N q),
$$

where $f(x)$ is a scaling function such that

$$
f(x) \sim\left\{\begin{array}{lll}
x^{-\alpha} & \text { if } & x \sim 1 \\
\text { const } & \text { if } & x \gg 1 .
\end{array}\right.
$$

The random network limit is obtained provided that

$$
\bar{d} \sim q^{\alpha(\bar{K})} N^{\gamma_{S w}(\bar{K})}(N q)^{-\alpha} \sim N^{\gamma_{\mathrm{Sw}}-\alpha} \sim N^{\gamma_{R}},
$$

i.e.,

$$
\gamma_{R}=\gamma_{\mathrm{Sw}}-\alpha(\bar{K}) .
$$

Given $\gamma_{R}$ we can express $\gamma_{\text {Sw }}$ by measuring $\alpha(\bar{K})$ at different $\bar{K}$. Figure 5 shows the reconstructed $\gamma_{S W}$ with the measured data, which satisfy the above proposed asymptotic scaling relation.

To analyze our data, Eq. (12) can also be written as

$$
\bar{d}_{\mathrm{SW}}(q, \bar{K}, N) \sim \frac{(N q)^{\gamma_{\mathrm{SW}}}}{q^{\gamma_{\mathrm{SW}}-\alpha}} f(N q) \sim \frac{1}{q^{\gamma_{R}}} g(N q),
$$

where $g(x)=x^{\gamma_{\text {Sw }}} f(x)$. Thus plotting $\bar{d} q^{\gamma_{R}}$ vs $N q$ should yield coinciding data with $g(x)$. The limits of random and spatially local networks correspond to the asymptotic small and large argument of $g(x)$, which gives us the exponents $\gamma_{R}$, and $\gamma_{L}$,

$$
g(x) \sim\left\{\begin{array}{lll}
x^{\gamma_{R}} & \text { if } \quad x \sim 1 \\
x^{\gamma_{S W}} \rightarrow x^{\gamma /} & \text { if } \quad x \gg 1 .
\end{array}\right.
$$

Figure 6 shows the scaling plots of the Hamming distance as a function of the product of the system size $N$ and strength of 


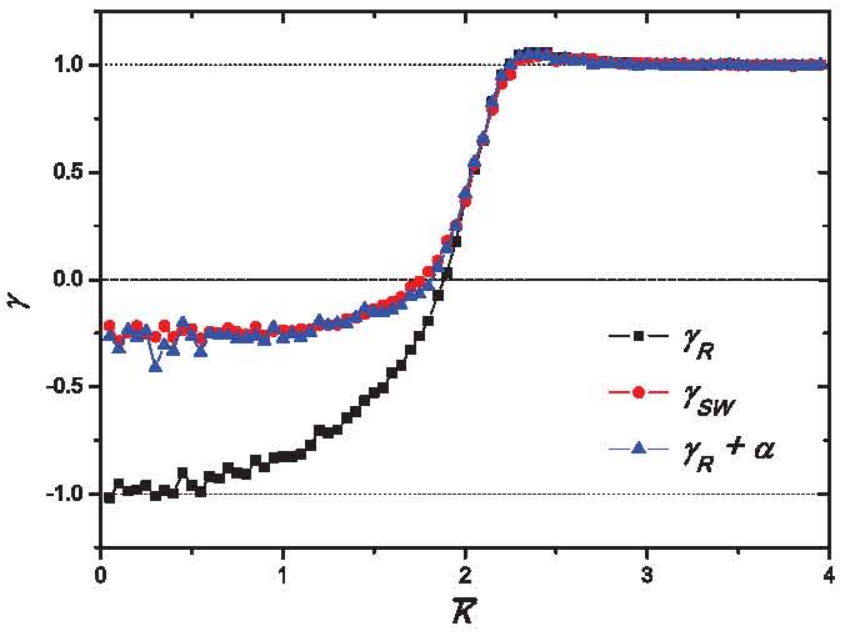

FIG. 5. (Color online) Reconstructed $\gamma_{\text {sw }}$ from Eq. (15) by measuring $\alpha(\bar{K})$ from best fits of Fig. 4 at $q=0.2(p=0.8)$. Squares (black) and circles (red) are measured $\gamma_{R}$ and $\gamma_{S w}$, respectively, from Fig. 1, while triangles (blue) are reconstructed $\gamma_{S w}$ from $\gamma_{R}$ and $\alpha(\bar{K})$.

the local connections $q$, as predicted by the proposed finite-size scaling for small-world RBNs. Also, given that $\gamma_{R}$ and $\gamma_{L}$ are functions of the average degree $\bar{K}$, as shown in Fig. 2, the shapes of $f(x)$ or $g(x)$ also change with $\bar{K}$. As one can see in Figs. 6(a)-6(c), $g(x)$ coincides under different $\bar{K}$. In addition, the asymptotic behavior of $g(x)$ at $x \sim 1$ and $x \gg$ 1 agrees very well with our measured "phenomenological" exponents $\gamma_{R}(\bar{K})$, and $\gamma_{L}(\bar{K})$ at $\bar{K}=1.4, \bar{K}=2.0$, and $\bar{K}=$ 4.0 , respectively.

\section{WIRING COST}

From an engineering perspective, one wants to typically minimize the wiring cost of a network, maximize the communication characteristics, and maximize the robustness against failures. The electric power grid is a good example and so are nanoscale interconnect networks [26]. In this section we will look at these three trade-offs for RBNs.

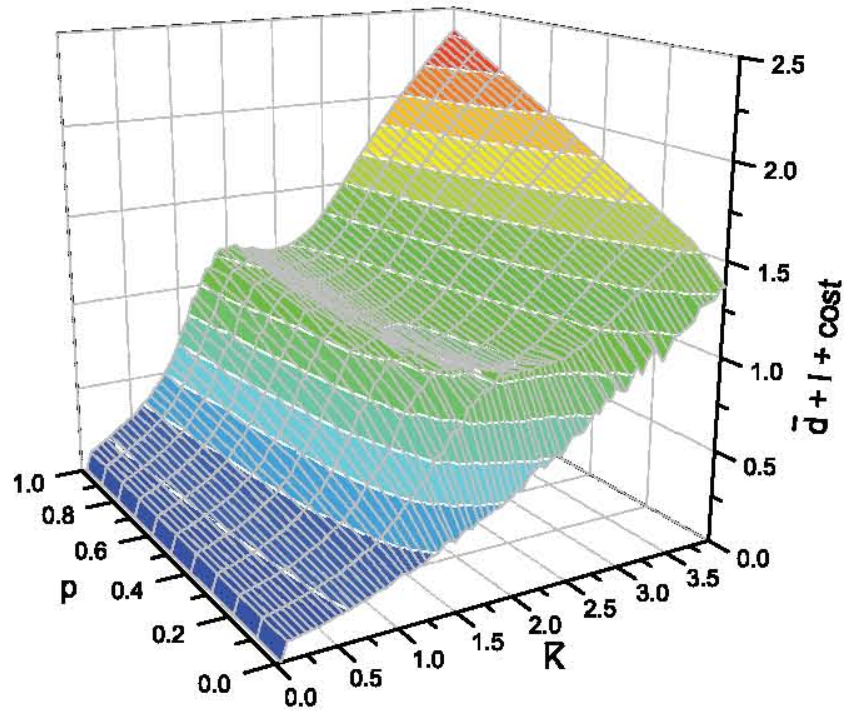

FIG. 7. (Color online) The product of damage size $d$, the network wiring cost "cost," and the average shortest path length $l$ as a function of the average connectivity $\bar{K}$ and the density of random connections $p$. The color density corresponds to the value of $d \times l \times$ cost. $d, l$, and cost were normalized.

The average shortest path length is generally a good measure for the communication characteristics of a complex network. In a directed network we define $l$ as the mean geodesic (i.e., shortest) distance between vertex pairs in a network [22]:

$$
l=\frac{1}{n(n-1)} \sum_{i \neq j} d_{i j}
$$

where $d_{i j}$ is the geodesic distance from vertex $i$ to vertex $j$. Here we have excluded the distance from each node to itself. Equation (18) will be problematic if the network has more than one component, which is very likely for small $\bar{K}$. To avoid the problem of disconnected networks, we compute the average
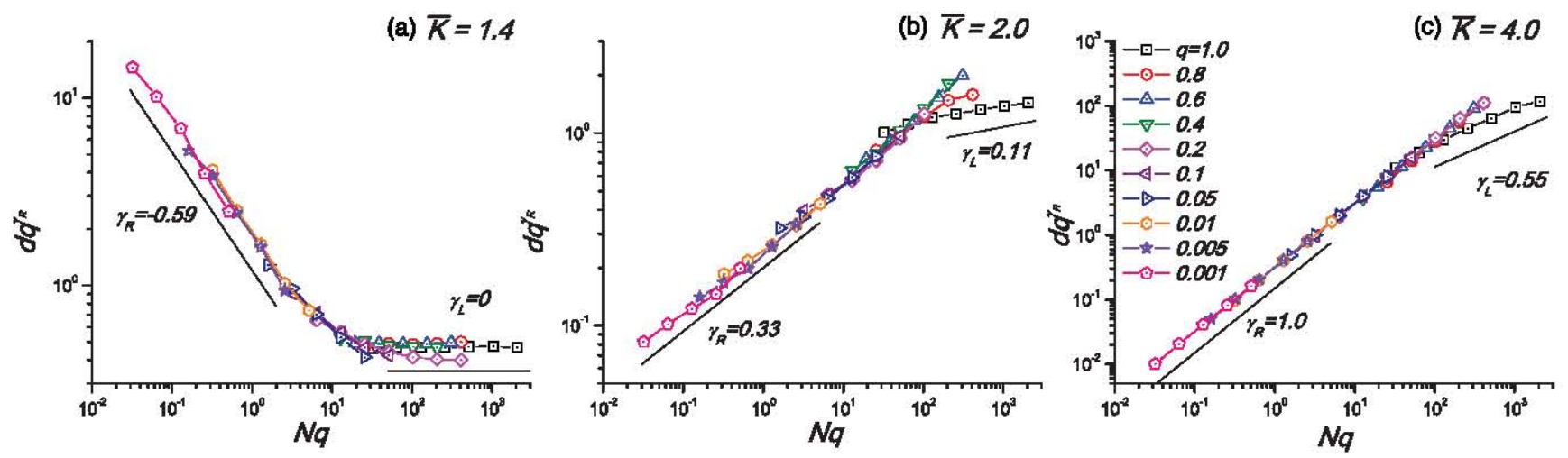

FIG. 6. (Color online) Scaling plots of the Hamming distance in small-world networks as predicted by the finite-size scaling argument [Eq. (16)]. (a) $\bar{K}=1.4$; (b) $\bar{K}=2.0$; and (c) $\bar{K}=3.0$. The straight line segments correspond to asymptotic power-law behavior of the scaling function $g(x)$ with exponents $\gamma_{R}$ and $\gamma_{L}$, measured from Fig. 2 at given $\bar{K}$, for small and large arguments, respectively, as described in the text [Eq. (17)]. 


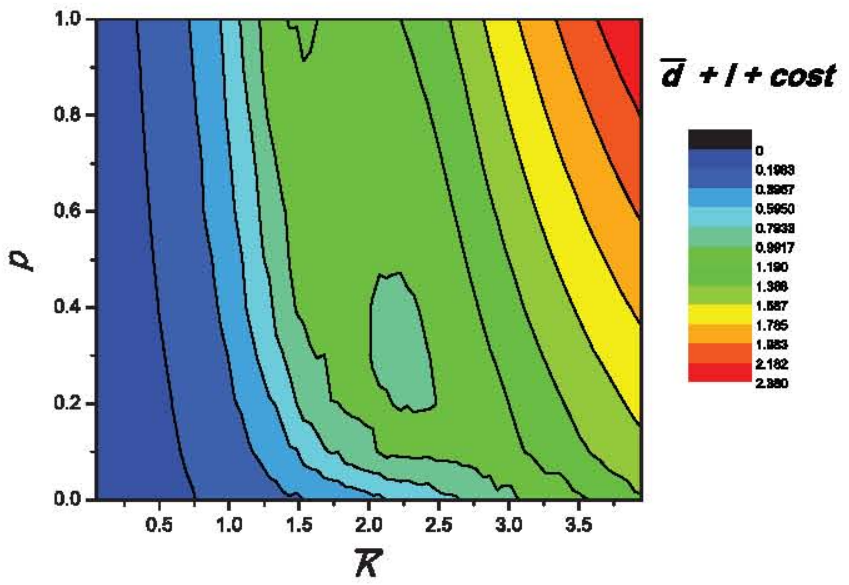

FIG. 8. (Color online) Contour projection of Fig. 7. The color density corresponds to the value of $d+l+\cos$. At $p=0$ the network topology is completely local; $p=1$ corresponds to the random network; and for $0<p<1$ the network is in the small-world regime. The circles indicate the position of lowest possible $\bar{K}$ and corresponding $p$ for given tolerance level of $d \times l \times \operatorname{cost} . d, l$, and cost were normalized.

path length only for those vertex pairs that actually have a connecting path between them.

For real-world networks, if shortcuts, i.e., the random small-world links, have to be realized physically, the cost of a long-range connection is likely to grow with its length. For example, the power consumption for wireless broadcast communication in free space generally could be a cubic power of geometric distance, while the implementation of directional antenna will reduce the transmission cost significantly [27]. Petermann et al. [28] discuss the wiring cost for some spatial small-world networks ranging from integrated circuits, the Internet to cortical networks. For simplicity, we assume here that the wiring cost has a linear dependency on the geometrical distance between two nodes.

Figure 7 shows the aggregate sum of the wiring cost, the average shortest path length $l$, and the damage size $\bar{d}$ as a function of the average connectivity $\bar{K}$ and the density of random connections $p$. For a given $\bar{K}$ and $p$, one can therefore find the network with the lowest cost, the best performance, and the highest robustness. The contour lines allow one to determine the optimal $\bar{K}$ and $p$ for a fixed aggregated sum.
In addition, Fig. 8 shows the $z$ projection of Fig. 7 using the same data. For example, it shows that for a given specific target average connectivity $\bar{K}$, networks with a high proportion of random connections (i.e., close to random networks, $p \rightarrow 1$ ) will have a similar overall performance, cost, and robustness as networks with more local connections $(p \rightarrow 0)$; however, whereas $p \rightarrow 1$ networks have a high wiring cost, a low average path length, and a low damage resistivity, $p \rightarrow 0$ networks have a low wiring cost, a high average path length, and a high damage resistivity.

\section{CONCLUSION}

We have systematically investigated the damage spreading in spatial and small-world random Boolean networks. We have found that (1) spatially local connections change the scaling of the Hamming distance at very low connectivities $(\bar{K} \ll 1)$ and (2) that the critical connectivity of stability $K_{s}$ changes compared to random networks [1]. Ribeiro et al. [29] reported similar results, namely, that by changing the local network structure, $K_{s}$ shifts. At higher $\bar{K}$, this scaling remains unchanged. We also show that the Hamming distance of spatially local networks scales with a power law as the system size $N$ increases, but with a different exponent for local and small-world networks. In addition, we have investigated the trade-offs between the wiring cost of the networks, the communication characteristics, and the robustness, i.e., the damage spreading. From an engineering perspective, one typically wants to minimize the wiring cost, maximize the communication characteristics, e.g., the shortest path between any two nodes, and maximize the robustness against failures. Our new findings provide these key trade-offs and allow one to determine the lowest connectivity $\bar{K}$ and the amount of randomness $p$ in a network for a given robustness, average path length, and wiring cost.

Future work will focus on the investigation of real-world networks and the application of our methodology to make them more robust, cheaper, and more efficient.

\section{ACKNOWLEDGMENTS}

We gratefully acknowledge the support of the US Department of Energy through the LANL/LDRD Program for this work. The authors thank Natali Gulbahce, Gyorgy Korniss, Thimo Rohlf, and Allen Taylor for their helpful comments on this work.
[1] T. Rohlf, N. Gulbahce, and C. Teuscher, Phys. Rev. Lett. 99, 248701 (2007).

[2] L. A. N. Amaral, A. Scala, M. Barthélémy, and H. E. Stanley, Proc. Natl. Acad. Sci. USA 97, 11149 (2000).

[3] S. A. Kauffman, J. Theor. Biol. 22, 437 (1969).

[4] S. A. Kauffman, The Origins of Order: Self-Organization and Selection in Evolution (Oxford University Press, New York, 1993).

[5] B. Derrida and Y. Pomeau. Europhys. Lett. 1, 45 (1986).

[6] R. Albert and A.-L. Barabási, Phys. Rev. Lett. 84, 5660 (2000).
[7] B. Samuelsson and C. Troein, Phys. Rev. Lett. 90, 098701 (2003).

[8] U. Bastola and G. Parisi, Physica D 115, 203 (1998).

[9] R. Pastor-Satorras and A. Vespignani, Phys. Rev. Lett. 86, 3200 (2001).

[10] M. E. J. Newman, Phys. Rev. E 66, 016128 (2002).

[11] R. Cohen, S. Havlin, and D. ben-Avraham, Phys. Rev. Lett. 91, 247901 (2003).

[12] M. L. Sachtjen, B. A. Carreras, and V. E. Lynch, Phys. Rev. E 61, 4877 (2000). 
[13] P. Rämö, J. Kesseli, and O. Yli-Harja, J. Theor. Biol. 242, 164 (2006).

[14] M. Paczuski, K. E. Bassler, and Á. Corral, Phys. Rev. Lett. 84, $3185(2000)$.

[15] R. Sole and B. Luque, Phys. Lett. A 196, 331 (1995).

[16] B. Luque and R. Sole, Phys. Rev. E 55, 257 (1997).

[17] V. Kaufman, T. Mihaljev, and B. Drossel, Phys. Rev. E 72, 046124 (2005).

[18] T. Mihaljev and B. Drossel, Phys. Rev. E 74, 046101 (2006).

[19] C. Darabos, M. Giacobini, and M. Tomassini, Adv. Complex Syst. 10, 85 (2007).

[20] B. Samuelsson and J. E. S. Socolar, Phys. Rev. E 74, 036113 (2006).

[21] D. J. Watts, Small Worlds: The Dynamics of Networks Between Order and Randomness (Princeton University Press, Princeton, NJ, 2003).
[22] M. E. J. Newman, SIAM Rev. 45, 167 (2003).

[23] B. Kozma, M. B. Hastings, and G. Korniss, Phys. Rev. Lett. 95, 018701 (2005).

[24] B. Kozma, M. B. Hastings, and G. Korniss, J. Stat. Mech.: Theory Exp. (2007) P08014.

[25] D. J. Watts and S. H. Strogatz, Nature (London) 393, 440 (1998).

[26] C. Teuscher, Chaos 17, 026106 (2007).

[27] Z. Huang, Z. Zhang, and B. Ryu, Proceedings of the 2006 International Conference on Wireless Communications and Mobile Computing, Vancouver, British Columbia, Canada (ACM, New York, 2006), pp. 917-922.

[28] T. Petermann and P. De Los Rios, Phys. Rev. E 73, 026114 (2006).

[29] A. S. Ribeiro, J. Lloyd-Price, J. Kesseli, A. Häkkinen, and O. Yli-Harja, Phys. Rev. E 78, 056108 (2008). 